\title{
A Prospective Study of Telomere Length Measured by Monochrome Multiplex Quantitative PCR and Risk of Lung Cancer
}

\author{
Min Shen ${ }^{1}$, Richard Cawthon ${ }^{2}$, Nathaniel Rothman ${ }^{1}$, Stephanie J. Weinstein ${ }^{1}$, Jarmo \\ Virtamo ${ }^{3}$, H. Dean Hosgood III ${ }^{1}$, Unhee Lim ${ }^{4}$, Demetrius Albanes ${ }^{1,{ }^{*}}$, and Qing Lan ${ }^{1, *}$ \\ ${ }^{1}$ Division of Cancer Epidemiology and Genetics, NCI, NIH, DHHS, Bethesda, MD 20892 \\ ${ }^{2}$ Department of Human Genetics, University of Utah, Salt Lake City, UT $84112{ }^{3}$ Department of \\ Chronic Disease Prevention, National Institute of Health and Welfare, Helsinki, Finland \\ ${ }^{4}$ Epidemiology Program, Cancer Research Center of Hawaii, Honolulu, HI 96813
}

\begin{abstract}
Purpose-Telomere length plays an important role in chromosomal stability and tumorigenesis, and its measurement in peripheral white blood cell DNA may be a predictor of the development of lung cancer.
\end{abstract}

Experimental Design-Using a new method - monochrome multiplex quantitative PCR -which reduces measurement variability, we compared telomere length relative to standard DNA in white blood cell DNA in 229 incident male lung cancer cases and 229 matched controls within the prospective Alpha-Tocopherol, Beta-Carotene Cancer Prevention (ATBC) Study of male smokers.

Results-Median $\left(10^{\text {th }}, 90^{\text {th }}\right.$ percentile) telomere length was $1.13(0.86,1.45)$ in cases and 1.08 $(0.85,1.38)$ in controls $(\mathrm{P}=0.038)$. Telomere length was inversely associated with pack-years of smoking (Spearman correlation $r=-0.16, \mathrm{P}=0.02$ ) among controls. Compared to subjects with shorter telomere length ( $\leq$ median), subjects with greater telomere length (> median) had a 1.6fold (95\% CI, 1.06-2.36) increased risk of lung cancer. There was a significant linear relationship between quartiles of telomere length and risk of lung cancer (odds ratios (95\% confidence intervals) by quartile: $1.00,0.98(0.55-1.73), 1.62(0.95-2.77)$, and $1.50(0.84-2.68) ; \mathrm{P}_{\text {trend }}=$ $0.05)$. In addition, subgroup analysis showed that greater telomere length was associated with increased risk of lung cancer among heavy smokers (> 38 years) $(\mathrm{OR}, 1.90 ; 95 \% \mathrm{CI}, 1.00-3.59)$ but not among light smokers ( $\leq 38$ years) $(\mathrm{OR}, 1.08 ; 95 \% \mathrm{CI}, 0.56-2.11)\left(\mathrm{P}_{\text {interaction }}=0.01\right)$.

Conclusions-Our results suggest that greater telomere length may be associated with higher risk of lung cancer among male smokers.

\section{Keywords}

Telomere length; lung cancer; cohort study

Authors for correspondence: Min Shen, M.D., Ph.D., Occupational \& Environmental Epidemiology Branch, Division of Cancer Epidemiology and Genetics, National Cancer Institute, NIH, DHHS, MSC 7240, 6120 Executive Blvd., EPS 8122, Bethesda, Maryland 20892-7240, Telephone: 301-451-8791, Fax: 301-402-1819, shenmi@ mail.nih.gov.

D.A. and Q.L. contributed equally to this work 


\section{Introduction}

Telomeres are tandem repeats of the sequence TTAGGG at the 3' end of chromosomes. They protect chromosomes from end-to-end fusions, misrepair and degradation (1). Telomere length is maintained by an enzyme called telomerase which is present in very low or undetectable concentrations in most normal human somatic cells. In telomerase-negative cells, telomeric DNA is progressively lost with cell divisions, and after reaching a critically short length, cells enter replicative senescence $(1 ; 2)$. As a result, shortening of telomeres is associated with human aging (3). In checkpoint defective cells, telomere dysfunction resulting from excessive telomere attrition or disruption of telomere structure may initiate chromosomal instability (1). However, short telomeres may suppress tumor formation by limiting the proliferation of transformed cells (4). Short telomeres have been related to both pro- and anti-tumor effects in mouse models (3).

Epidemiological studies are inconsistent about the association between telomere length and cancer risk. Some investigations indicate that relatively shorter telomere length measured in peripheral white blood cells, and in some instances buccal cells, is associated with increased risk of lung cancer, head and neck cancer, renal cell carcinoma, bladder cancer, esophageal adenocarcinoma, and non-Hodgkin lymphoma (5-11). In contrast, some recent reports have suggested that greater telomere length may be associated with higher risk of breast cancer and melanoma (12;13). Most of these studies were of retrospective case-control design with blood samples being collected after the cases were diagnosed or treated $(5 ; 6 ; 9-11)$. We tested the hypothesis that altered telomere length in peripheral white blood cell DNA might be associated with risk of developing lung cancer in a prospective cohort, the AlphaTocopherol, Beta-Carotene Cancer Prevention (ATBC) Study (14).

\section{Materials and methods}

Details of the ATBC Study design have been described previously (14). Briefly, 29,133 male smokers, aged 50-69 years, were recruited from 1985 through 1988 from southwest Finland and randomized to receive either $a$-tocopherol, $\beta$-carotene, both $\alpha$-tocopherol and $\beta$-carotene, or neither of them. The study was approved by Institutional Review Boards at the National Cancer Institute and the National Public Health Institute of Finland. All participants provided written informed consent. Incident lung cancer cases were identified using the Finnish Cancer Registry, which provides nearly $100 \%$ of case ascertainment in Finland (15).

Incident lung cancer cases ( $\mathrm{n}=229)$ diagnosed through April, 2003 were randomly selected from among participants that provided a whole blood sample in 1992 or 1993. As a result of more detailed case review during the ATBC Trial period, fewer than $50 \%$ of cases had clear classification as squamous cell carcinoma $(n=74)$ or adenocarcinoma $(n=34)$. Another 79 cases were of epithelial origin without further classification. Controls were randomly selected from the ATBC cohort members who were alive, free of cancer at the time of the case's diagnosis, and were individually matched to cases on date of birth ( \pm 5 years).

DNA was extracted from whole blood using the phenol-chloroform method, and a monochrome quantitative multiplex PCR assay was used to measure the relative telomere length (16). In brief, the reagents in the 25 microliter PCR were $10 \mathrm{mM}$ Tris- $\mathrm{HCl} \mathrm{pH} \mathrm{8.3,50}$ $\mathrm{mM} \mathrm{KCl}, 3 \mathrm{mM} \mathrm{MgCl} 2,0.2 \mathrm{mM}$ each dNTP, $1 \mathrm{mM}$ DTT, $1 \mathrm{M}$ betaine, $0.75 \mathrm{x}$ SYBR Green I, and AmpliTaq Gold DNA polymerase, 0.625 U. Four primers were used $\left(5^{\prime}\right.$ to $\left.3^{\prime}\right)$ : telg (at $900 \mathrm{nM}$ ), telc (at $900 \mathrm{nM}$ ), hbgu (at $500 \mathrm{nM}$ ), and hbgd (at $500 \mathrm{nM}$ ). Three-fold serial dilutions of a reference genomic DNA sample were used to generate two standard curves for each PCR plate. After the run was complete, the MyiQ software (Bio-Rad iQ5 2.0 Standard Edition Optical System Software) was used to determine the T (telomere) and S (single copy 
gene) values for each experimental sample by the Standard Curve method. T/S, therefore, is a relative and dimensionless value which is proportional to the average telomere length per cell. Samples with a T/S of $>1.0$ have an average telomere length greater than that of the standard DNA; samples with a T/S of $<1.0$ have an average telomere length shorter than that of the standard DNA. This multiplex quantitative PCR method eliminates the pipetting variation between wells present in monoplex quantitative PCR (16).

Cases and their matched controls were blindly assayed consecutively within each batch along with quality control duplicate samples (5\%) that were interspersed in each batch to evaluate assay reproducibility. Samples were analyzed once or in duplicate. The intra-class correlation coefficient of the assay was $80 \%$ and the overall coefficient of variation was $7 \%$.

Correlation between age, pack-years of smoking and relative telomere length was determined with the Spearman correlation. Telomere length and pack-years of smoking were categorized into two and four categories, respectively, based on median and quartiles among controls. Odds ratios (OR) and 95\% confidence intervals (95\% CI) were estimated using conditional logistic regression models. Tests for trend were calculated by treating the level of telomere length as a continuous variable. The model was adjusted for age, number of cigarettes per day, and number of years smoked. No other factors, including body mass index, smoking, physical activity, dietary intake, blood pressure, and mitochondrial DNA copy number, were found to introduce a $\geq 10 \%$ change in the beta-coefficient for telomere length in the base model. Interactions between telomere length and factors including years of smoking, cigarettes per day, age, and $\alpha$-tocopherol or $\beta$-carotene supplementation were tested by inserting a cross-product interaction term between them in the model. Subgroup analysis was conducted to calculate effect of telomere length by factors with significant interaction with telomere length. All data were analyzed using SAS 9.1.3 (SAS Institute Inc, Cary, NC).

\section{Results}

Cases were comparable to controls with respect to age and alcohol consumption (Table 1). Average BMI was somewhat lower for cases than controls $\left(\mathrm{P}_{\mathrm{t} \text {-test }}=0.04\right)$. Even though all subjects in the cohort smoked cigarettes at the time they were enrolled into the study, lung cancer cases smoked more cigarettes per day for a longer period of time than controls $\left(\mathrm{P}_{\mathrm{t} \text {-test for pack-year }}=0.001\right)$. When categorized by quartiles of pack-years, heavy smokers had the highest risk of developing lung cancer $\left(\mathrm{OR}, 2.57 ; 95 \% \mathrm{CI}, 1.49-4.45, \mathrm{P}_{\text {trend }}=0.0002\right)$ (Table 2).

Telomere length was inversely and non-significantly associated with age (Spearman correlation $r=-0.09, \mathrm{P}=0.18$ ) among controls, which could be explained by the relatively narrow age range of this cohort of tobacco smokers. Telomere length was also inversely associated with pack-years of smoking (Spearman correlation $r=-0.16, \mathrm{P}=0.02$ ) among controls. Telomere length was comparable in the trial a-tocopherol or $\beta$-carotene supplementation arms.

Telomere length was statistically significantly greater among cases than among controls (median, $10^{\text {th }}, 90^{\text {th }}$ percentile $1.13,0.86,1.45$ in cases and $1.08,0.85,1.38$ in controls; $\mathrm{P}_{\text {t-test }}$ $=0.038$ ). The risk of lung cancer for men with greater than median telomere length was significantly higher than for those with shorter telomeres (OR, 1.59; 95\% CI, 1.06-2.36). When the telomere length was categorized by quartiles, we observed a significant linear trend $(\mathrm{P}=0.05)$ for increased lung cancer risk and greater telomere length, even though the ORs for specific quartiles were only of borderline statistical significance (Table 2). 
To determine if the telomere length - lung cancer association might be driven in part by undiagnosed lung cancer cases at the time of blood collection, we excluded the cases diagnosed within the first year of follow-up $(\mathrm{n}=14)$ after blood sample collection, and found that results were very similar, with an OR of 1.56 for subjects with higher than median telomere length compared with subjects with shorter telomeres (95\% CI, 1.02-2.38). Subgroup analyses of cases diagnosed in different periods after blood collection $(0-3,3-5$, $5-8$, and $>8$ years) showed similar but non-significant results (data not shown).

Subgroup analyses restricted to lung cancer of epithelial origin $(n=187)$ and squamous cell carcinoma (the only histology subgroup with enough cases for conditional logistic regression) obtained comparable but non-significant results compared with the overall analysis (data not shown). Analyses stratified by the trial $\alpha$-tocopherol or $\beta$-carotene supplementation arms found risks to be similar across the intervention groups, and test for interaction was not statistically significant (data not shown).

A significant positive interaction was found between telomere length and years of smoking $(P=0.01)$. Subgroup analysis by median of years of smoking in controls showed that greater telomere length was associated with increased risk of lung cancer among subjects of $>38$ years of smoking (OR, 1.90; 95\% CI, 1.00-3.59) but not among subjects of $\leq 38$ years of smoking (OR, 1.08; 95\% CI, 0.56-2.11) (Table 3). Heavy smokers with longer telomere length had highest risk of lung cancer (OR, 2.88; 95\% CI, 1.60-5.21) (Figure 1).

\section{Discussion}

To the best of our knowledge, this is the first report of a positive association between telomere length measured in white blood cells and risk of lung cancer, particularly among heavy smokers, in a prospective cohort study. Telomere length in different tissues of the same people is correlated and therefore telomere length measured in white blood cells could serve as a surrogate parameter for telomere length in other tissues (17). These findings provide novel insight into the role of the telomere in lung cancer pathogenesis.

Telomere shortening was observed first in malignant tumor tissue (2). It was thereafter considered a risk factor for cancer initiation and development. However, both shorter and longer telomeres can predispose to the development of cancer from a theoretical perspective in the context of the cell microenvironment and potential impact on senescence or apoptosis (18). Optimal telomere length is achieved through the balance of cell proliferation, senescence, and apoptosis. Shorter telomeres may lead to chromosomal instability, which could reflect a constitutional or acquired tendency towards carcinogenesis $(9 ; 11)$. On the other hand, maintaining long telomeres for longer periods may facilitate tumorigenesis by predisposing cells to delayed senescence and greater risk of opportunity for acquiring genetic abnormalities.

In addition, the relationship between telomere length and risk of cancer may vary by cell type, dependent on the proliferation potential. Han and his colleagues reported from a cohort study that telomere length was positively associated with risk of melanoma, but negatively associated with risk of basal cell carcinoma (12). This may reflect the different routes of tumorigenesis of melanoma and basal cell carcinoma.

In contrast to our results, short telomere length was found to be associated with higher risk of lung cancer in two case-control studies $(6 ; 11)$. The discrepancy between these and the present findings may be due to the use of retrospective versus prospective study designs or the possible effects of cancer therapies initiated among some of the cases enrolled in the retrospective studies. For example, tumor chemotherapy has been shown to result in shorter telomere length in peripheral blood cells $(19 ; 20)$. Finally, selection bias may happen in case- 
control study and impact the comparison between cases and controls. Our controls were selected randomly and samples were collected before the diagnosis and treatment of any cancer. Cases and controls are comparable on age and we also adjusted for age in regression model to control additional possible confounding.

Our finding is restricted to heavy smokers; however, telomere length was inversely associated with pack-year of smoking. It may imply that shortening telomeres is not the intermediate factor for the carcinogenic effect of smoking on lung cancer. Tobacco smoking is not only a confounding factor but also an effect-measure modifier. Our findings suggest that heavy smokers who manage to maintain telomere length are at higher risk for lung cancer.

One restriction of our study is that the assay is based on DNA extracted from a mixture of various types of white blood cells. It is known that telomere length may be different among major blood cell types $(21 ; 22)$. Our cases smoked more than the controls. Cases may have a higher chance to have inflammation than controls, and thus the composition of blood cells (granulocytes, lymphocytes, monocytes, etc) may be different between cases and controls. We checked one important biomarker of inflammation, C-reactive protein (CRP), among a subset of the ATBC cohort members. The CRP is not associated with cigarette per day $(\mathrm{p}=0.25)$, years of smoking $(\mathrm{p}=0.06)$, and pack-years of smoking $(\mathrm{p}=0.64)$ in healthy controls $(n=221)$ (unpublished data). The 15 cases and 36 controls that overlapped with the current telomere length study had comparable CRP levels ( $\mathrm{p}=0.85$ ). In addition, blood samples were withdrawn before diagnosis of cancer and cases and controls are comparable in age. Therefore, the composition of blood cells is unlikely to be systematically different between cases and controls.

In summary, we found that greater relative telomere length was associated with an increased subsequent risk of lung cancer in male smokers. The relationship between telomere length and risk of cancer may vary by cancer type. These findings need to be replicated by other studies using designs that avoid potential biases from sample type, therapy, and assay methods.

\section{Acknowledgments}

This work was supported by intramural funds from the National Cancer Institute and extramural funds, including U.S. Public Health Service contracts N01-CN-45165, N01-RC-45035, and N01-RC-37004 from the National Cancer Institute, National Institutes of Health, Department of Health and Human Services.

\section{References}

1. Cheung AL, Deng W. Telomere dysfunction, genome instability and cancer. Front Biosci. 2008; 13:2075-90. [PubMed: 17981693]

2. Raynaud CM, Sabatier L, Philipot O, Olaussen KA, Soria JC. Telomere length, telomeric proteins and genomic instability during the multistep carcinogenic process. Crit Rev Oncol Hematol. 2008; 66:99-117. [PubMed: 18243729]

3. Londono-Vallejo JA. Telomere instability and cancer. Biochimie. 2008; 90:73-82. [PubMed: 17728038]

4. Djojosubroto MW, Choi YS, Lee HW, Rudolph KL. Telomeres and telomerase in aging, regeneration and cancer. Mol Cells. 2003; 15:164-75. [PubMed: 12803478]

5. Broberg K, Bjork J, Paulsson K, Hoglund M, Albin M. Constitutional short telomeres are strong genetic susceptibility markers for bladder cancer. Carcinogenesis. 2005; 26:1263-71. [PubMed: 15746160]

6. Jang JS, Choi YY, Lee WK, et al. Telomere length and the risk of lung cancer. Cancer Sci. 2008; 99:1385-9. [PubMed: 18452563] 
7. McGrath M, Wong JY, Michaud D, Hunter DJ, De VI. Telomere length, cigarette smoking, and bladder cancer risk in men and women. Cancer Epidemiol Biomarkers Prev. 2007; 16:815-9. [PubMed: 17416776]

8. Risques RA, Vaughan TL, Li X, et al. Leukocyte telomere length predicts cancer risk in Barrett's esophagus. Cancer Epidemiol Biomarkers Prev. 2007; 16:2649-55. [PubMed: 18086770]

9. Shen J, Terry MB, Gurvich I, et al. Short telomere length and breast cancer risk: a study in sister sets. Cancer Res. 2007; 67:5538-44. [PubMed: 17545637]

10. Widmann TA, Herrmann M, Taha N, Konig J, Pfreundschuh M. Short telomeres in aggressive non-Hodgkin's lymphoma as a risk factor in lymphomagenesis. Exp Hematol. 2007; 35:939-46. [PubMed: 17533048]

11. Wu X, Amos CI, Zhu Y, et al. Telomere dysfunction: a potential cancer predisposition factor. J Natl Cancer Inst. 2003; 95:1211-8. [PubMed: 12928346]

12. Han J, Qureshi AA, Prescott J, et al. A Prospective Study of Telomere Length and the Risk of Skin Cancer. J Invest Dermatol. 2008

13. Svenson U, Nordfjall K, Stegmayr B, et al. Breast cancer survival is associated with telomere length in peripheral blood cells. Cancer Res. 2008; 68:3618-23. [PubMed: 18483243]

14. The ATBC Cancer Prevention Study Group. The alpha-tocopherol, beta-carotene lung cancer prevention study: design, methods, participant characteristics, and compliance. The ATBC Cancer Prevention Study Group. Ann Epidemiol. 1994; 4:1-10. [PubMed: 8205268]

15. Korhonen P, Malila N, Pukkala E, et al. The Finnish Cancer Registry as follow-up source of a large trial cohort--accuracy and delay. Acta Oncol. 2002; 41:381-8. [PubMed: 12234031]

16. Cawthon RM. Telomere length measurement by a novel monochrome multiplex quantitative PCR method. Nucleic Acids Res. 2009; 37:e21. [PubMed: 19129229]

17. Friedrich U, Griese E, Schwab M, et al. Telomere length in different tissues of elderly patients. Mech Ageing Dev. 2000; 119:89-99. [PubMed: 11080530]

18. Mooi WJ, Peeper DS. Oncogene-induced cell senescence--halting on the road to cancer. N Engl J Med. 2006; 355:1037-46. [PubMed: 16957149]

19. Lee JJ, Nam CE, Cho SH, et al. Telomere length shortening in non-Hodgkin's lymphoma patients undergoing chemotherapy. Ann Hematol. 2003; 82:492-5. [PubMed: 12910376]

20. Yoon SY, Sung HJ, Park KH, et al. Telomere length shortening of peripheral blood mononuclear cells in solid-cancer patients undergoing standard-dose chemotherapy might be correlated with good treatment response and neutropenia severity. Acta Haematol. 2007; 118:30-7. [PubMed: 17429195]

21. Hoffmann J, Erben Y, Zeiher AM, Dimmeler S, Spyridopoulos I. Telomere length-heterogeneity among myeloid cells is a predictor for chronological ageing. Exp Gerontol. 2009; 44:363-6. [PubMed: 19248826]

22. Spyridopoulos I, Erben Y, Brummendorf TH, et al. Telomere gap between granulocytes and lymphocytes is a determinant for hematopoetic progenitor cell impairment in patients with previous myocardial infarction. Arterioscler Thromb Vasc Biol. 2008; 28:968-74. [PubMed: 18276909] 




Figure 1.

Figure Joint effect of smoking and telomere length on lung cancer risk. 
Table 1

Comparison of characteristics of lung cancer cases and individually-matched controls.

\begin{tabular}{|c|c|c|c|c|}
\hline \multirow{2}{*}{ Characteristic } & \multicolumn{2}{|c|}{ Cases $(n=229)$} & \multicolumn{2}{|c|}{ Controls $(n=229)$} \\
\hline & Mean (std) & Median & Mean (std) & Median \\
\hline Mean age at baseline, years & $59(5)$ & 59 & $58(5)$ & 58 \\
\hline \multicolumn{5}{|l|}{ Smoking } \\
\hline Number cigarettes/day & $21(8)$ & 20 & $19(8)$ & 20 \\
\hline Years of smoking & $38(7)$ & 40 & $36(9)$ & 38 \\
\hline Pack-years & $40(16)$ & 40 & $34(18)$ & 33 \\
\hline Body mass index, $\mathrm{kg} / \mathrm{m}^{2}$ & $26(3)$ & 25 & $26(4)$ & 26 \\
\hline Alcohol, g/day & $17(18)^{1}$ & $11^{1}$ & $18(20)^{2}$ & $13^{2}$ \\
\hline \multicolumn{5}{|l|}{ Supplementation Group } \\
\hline Placebo, $\%$ & 23 & & 29 & \\
\hline Alpha Tocopherol, \% & 25 & & 24 & \\
\hline Beta-Carotene, $\%$ & 26 & & 24 & \\
\hline Alpha Tocopherol/Beta-Carotene, $\%$ & 26 & & 23 & \\
\hline Telomere length & $1.14(0.23)$ & 1.13 & $1.10(0.22)$ & 1.08 \\
\hline
\end{tabular}


Table 2

Odds ratio (OR) and 95\% confidence interval (95\% CI) for smoking and telomere length and lung cancer risk.

\begin{tabular}{|c|c|c|c|c|}
\hline & Cases $(\%)(n=229)$ & Controls (\%) $(\mathrm{n}=229)$ & OR & $95 \% \mathrm{CI}$ \\
\hline \multicolumn{5}{|c|}{ Pack-years of smoking } \\
\hline 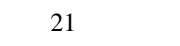 & $37(16)$ & $61(27)$ & $1.00^{1}$ & \\
\hline$>21-33$ & $40(17)$ & $57(25)$ & 1.20 & $0.67-2.13$ \\
\hline$>33-44$ & $65(28)$ & $58(25)$ & 1.92 & $1.09-3.39$ \\
\hline \multirow[t]{2}{*}{$>44$} & $87(38)$ & $53(23)$ & 2.57 & $1.49-4.45$ \\
\hline & & & & $0.0002^{2}$ \\
\hline \multicolumn{5}{|c|}{ Telomere length (by median) } \\
\hline$\unlhd 1.08$ & $91(40)$ & $115(50)$ & $1.00^{3}$ & \\
\hline$>1.08$ & $138(60)$ & $114(50)$ & 1.59 & $1.06-2.36$ \\
\hline \multicolumn{5}{|c|}{ Telomere length (by quartile) } \\
\hline$\unlhd) .94$ & $43(19)$ & $58(25)$ & $1.00^{3}$ & \\
\hline$>0.94-1.08$ & $48(21)$ & $57(25)$ & 0.98 & $0.55-1.73$ \\
\hline$>1.08-1.25$ & $72(31)$ & $57(25)$ & 1.62 & $0.95-2.77$ \\
\hline \multirow[t]{2}{*}{$>1.25$} & $66(29)$ & $58(25)$ & 1.50 & $0.84-2.68$ \\
\hline & & & & $0.05^{2}$ \\
\hline \multicolumn{5}{|c|}{ Model adjusted for age at randomization; } \\
\hline
\end{tabular}






